\title{
Phonon Dispersion of Alkali Metals Using the TB-SMA Model of Cohesion
}

\begin{abstract}
Aigbekaen E.E.,
Samuel Okunzuwa,

Department of Physics, College of Natural and Applied Sciences,

Igbinedion University Okada, Nigeria

Arthur I. Ejere,

Department of Physics, Faculty of Physical Sciences,

University of Benin, Benin City, Nigeria

Doi: 10.19044/esj.2019.v15n15p487 URL:http://dx.doi.org/10.19044/esj.2019.v15n15p487

Abstract

The determination of Phonon dispersion of alkali metals was unsuccessful using the parameter $n=1 / 2$ or $2 / 3$ in the past two decades. In this study the parameter $\mathrm{n}$ has been consistently determined for the different alkali metals, the force constant has been determined up to the fourth next nearest neighbour. The result showed that the TB-SMA dispersion curves were in good agreement with experiment. This good agreement with experiment of the TB-SMA Phonons counters the prevailing notion in the literature that the TB-SMA model fails for alkali metals.
\end{abstract}

Keywords: Phonon, Tight-Binding, Second- Moment- Approximation, Alkali and Dispersion

\section{Introduction}

An efficient and accurate method of calculating the total energies is desirable for the study of methods of interest to the materials scientist. Due to the enormous computation burden posed by these methods, empirical potentials, that include many-body interaction terms, beyond a pair potential term, have been developed [Daw and Baskes, (1984), Cleri and Rosato, (1993)]. These semi-empirical approaches have been found to provide fairly accurate results with considerable increased computational efficiency compared to ab-initio methods [Daw and Hatcher, (1985),Ozdemir et al, (2008)] or fast self consistent calculation techniques which are the ideal theoretical framework for handling these systems. In general, semi-empirical approaches tackle the many-body problem by 
determining a functional form for the cohesive energy based on some physical model [Jun and Jian-Sheng, (2002), Idiodi, (2000)].

The main advantage of a many-body potential treatment over the traditional and practically simple pair-potential treatment is the ability to better reproduce some basic features of metallic systems. Once these parameters have been determined, the functional form may then be used to calculate other properties such as defect energies.

Another test of the TB-SMA model potential is the calculation of phonon dispersion curves obtained by diagonalization of the dynamical matrix in the purely harmonic approximation [Guevara et al, (1995), Carlsson, (1991), Johnson, (1988)].

\section{Materials and Methodology}

\subsection{The Basic Equations of the EAM}

Idiodi [Idiodi, (2000)] high-lighted that the TB-SMA scheme is formally analogous to the embedded-atom method (EAM). Empirical potentials such as the EAM also describe the atomic interactions by several analytical functions

However, we give below the seven (7) basic equations that regularly arise when working practically with the EAM for BCC metals are as follows:

$$
\begin{aligned}
& U_{o}=4 \phi_{1}\left(r_{o}\right)+F\left(\rho_{e}\right) \\
& 0=4 \phi_{1}^{\prime}\left(r_{o}\right)+F^{\prime}\left(\rho_{e}\right)\left[\frac{V_{11}}{a}\right] \\
& 6 a B_{o}=4 \phi_{1}^{\prime \prime}\left(r_{o}\right)+F^{\prime \prime}\left(\rho_{e}\right)\left[\rho^{\prime}\left(r_{o}\right)\right]^{2}+F^{\prime}\left(\rho_{e}\right)\left[\rho^{\prime \prime}\left(r_{o}\right)\right] \\
& a C_{11}=\left[\frac{-4 \phi_{1}^{\prime}\left(r_{o}\right)}{3 \sqrt{3} a}+\frac{2}{3} \phi^{\prime \prime}\left(r_{o}\right)\right]+\frac{a F^{\prime}\left(\rho_{e}\right)}{\Omega_{o}} W_{11}+\frac{a F^{\prime \prime}\left(\rho_{e}\right)}{\Omega_{o}} V^{2}{ }_{11} \\
& a C_{12}=\left[\frac{-4 \phi_{1}^{\prime}\left(r_{o}\right)}{3 \sqrt{3} a}+\frac{2 \phi_{1}^{\prime \prime}\left(r_{o}\right)}{3}\right]+\frac{a F^{\prime}\left(\rho_{e}\right)}{\Omega_{o}} W_{12}+\frac{a F^{\prime \prime}\left(\rho_{e}\right)}{\Omega_{o}} V_{11}{ }^{2} \\
& a C_{44}=\left[\frac{-4 \phi_{1}^{\prime}\left(r_{o}\right)}{3 \sqrt{3} a}+\frac{2 \phi_{1}^{\prime \prime}\left(r_{o}\right)}{3}\right]+\frac{a F^{\prime}\left(\rho_{e}\right)}{\Omega_{o}} W_{12} \\
& E_{I V}^{F}=8 F\left(\frac{7 \rho_{e}}{8}\right)-7 F\left(\rho_{e}\right)-U_{o}
\end{aligned}
$$

In the equations above, $\mathrm{U}_{\mathrm{o}}$ is the equilibrium energy per atom, $\mathrm{B}_{\mathrm{o}}$ and $\mathrm{C}_{\mathrm{ij}}$ are, respectively, the bulk modulus, and elastic constants written in Voigt 
notation. $\Omega_{0}$ is the volume per atom in the solid, $r_{o}$ is the equilibrium nearestneighbour distance, and $\mathrm{a}$ is the equilibrium lattice constant.

$\phi_{1}$ is a repulsive pair potential whose first and second derivatives with respect to the radial distance $\mathrm{r}$ are respectively, $\phi_{1}^{\prime}$ and $\phi_{1}^{\prime \prime}$ ( all quantities being evaluated at $\mathrm{r}=\mathrm{r}_{\mathrm{o}}$ ), while $\mathrm{F}$ is the embedding function with respect to the density, $\rho$, with $F^{\prime}$ and $F^{\prime \prime}$ being the first two derivatives; again all quantities being evaluated at the equilibrium density $\rho_{e} \cdot E_{I V}^{F}$ is the mono vacancy formation energy, while $\mathrm{V}_{11}, \mathrm{~W}_{11}$ and $\mathrm{W}_{12}$ are EAM parameters.

In all the previous applications of the EAM, $\mathrm{V}_{11}, \mathrm{~W}_{11}$ and $\mathrm{W}_{12}$ have been considered as free parameters to be consistently determined so that the basic equations of the EAM are satisfied. Such an approach enables us to surmount the problem of the elastic constants not being correctly reproduced theoretically. Here, we shall introduce a slightly different approach since it is often desirable to predict several physical quantities from a minimum set of parameters.

For BCC lattice, $\mathrm{r}_{\mathrm{o}}=\frac{a(\sqrt{3})}{2}, \Omega_{o}=\frac{a^{3}}{2}$ and expressions for $\mathrm{V}_{11}, \mathrm{~W}_{11}$ and $\mathrm{W}_{12}$ in terms of the derivatives of the density are in the form

$$
V_{11}=r_{o} \rho^{\prime}\left(r_{o}\right) / 3
$$

It is obvious from equation (2.1) to (2.7) above that the EAM has three basic functions, i.e.; $\phi_{1}(r), F(\rho)$ and $\rho(r)$ which generate the 8 EAM parameters: $\phi_{1}\left(r_{o}\right), \phi_{1}^{\prime}\left(r_{o}\right), \phi_{1}^{\prime \prime}\left(r_{o}\right), F\left(\rho_{e}\right), F^{\prime}\left(\rho_{e}\right), F^{\prime \prime}\left(\rho_{e}\right), \rho^{\prime}\left(r_{o}\right)$, and $\rho^{\prime \prime}\left(r_{o}\right)$. All other parameters such as $\mathrm{V}_{11}, \mathrm{~W}_{11}$ and $\mathrm{W}_{12}$ are dependent on the 8 EAM parameters.

\subsection{The Basic Functions of the TB-SMA Scheme}

In an obvious correspondence with the EAM scheme, the expressions for the cohesive energy $U_{0}$, in the TB-SMA scheme is the sum of the two terms [Mehl and Papacontantopoulos, (1996), Karolewski, (2001), Idiodi and Aghemenloh, (1998), Willaime and Massobrio, (1989)]

$$
\mathrm{U}_{\mathrm{o}}=\mathrm{E}_{\mathrm{R}}+\mathrm{E}_{\mathrm{B}}
$$

The functions $E_{R}$ and $E_{B}$ are usually written in the form

$$
\mathrm{E}_{\mathrm{R}}=\mathrm{A} \sum_{j} \exp \left[-p\left(\frac{r_{i j}}{r_{o}}-1\right)\right]
$$


And

$$
\mathrm{E}_{\mathrm{B}}=-\xi\left\{\sum_{j} \exp \left[-2 q\left(\frac{r_{i j}}{r_{o}}-1\right)\right]\right\}^{n}
$$

It is clear that

$$
\rho\left(r_{i j}\right)=\left\{\sum_{j} \exp \left[-2 q\left(\frac{r_{i j}}{r_{o}}-1\right)\right]\right\}^{n}
$$

Density as a function of the atomic distance ij.

Equations (2.10), (2.11), and (2.12) are the equations for the three basic functions of the TB-SMA scheme.

The sum over $\mathrm{j}$ in equations (2.10) to (2.12) is actually a sum over neighbours, $r_{i j}$, being the distance between atoms $i$ and $j$ and $r_{o}$ is the bulk nearest neighbour distance.

The TB-SMA expressions for the functions $\cdot \phi_{1}(r), F(\rho)$ and $\rho(r)$ as contained in the equations (2.9) to (2.12) are governed by only five (5) parameters (A, p, $\xi, \mathrm{q}$, and $\mathrm{n})$, instead of the eight (8) parameters $\left(\phi_{1}\left(r_{o}\right), \phi_{1}^{\prime}\left(r_{o}\right), \phi_{1}^{\prime \prime}\left(r_{o}\right), F^{\prime}\left(\rho_{e}\right), F^{\prime \prime}\left(\rho_{e}\right), \rho^{\prime}\left(r_{o}\right)\right.$ and $\left.\rho^{\prime \prime}\left(r_{o}\right)\right)$ which are encountered in the EAM scheme (Equations (2.1) to (2.7)). In the initial development of the TB-SMA scheme, the five (5) basic parameters were determined by fitting results from ab initio calculations for high symmetry structures, the only experimental input being the lattice constant. The fitting procedure is quite cumbersome since the ab initio results are not easily available. Physical quantities like the elastic constants, the vacancy formation energy, etc. were subsequently predicted by the theory. Unfortunately, this ambitious programme [Sigalas and Papaconstantopoulos, (1991), Strich et al, (1991)] is yet to reach the much desired perfection stage, since several predicted quantities are in conflict with experiment.

In several applications of the TB -SMA scheme the parameter $n$ is specified. In this situation the four remaining free parameters of the TB-SMA scheme can be determined from the EAM equations either using equations (2.1), (2.2), (2.3), and (2.7) or equations (2.1), (2.2), (2.3) and any one of (2.4) to $(2.6)$.

The determined TB-SMA parameter $\mathrm{p}$, for the BCC metals (see Table 1 ), though is very large compared to typical values in the literature [Li et al, (2003)] satisfy the formulas

$$
\begin{aligned}
\xi & =\frac{p E_{c}}{(p-q) \sqrt{2}} \quad \text { and } \\
A & =\frac{q E_{c}}{(p-q) z}
\end{aligned}
$$


given by Cleri and Rosato (1993).

where $E_{c}$ is the cohesive energy and $z$ is the coordination number for Alkali metals.

In practice, determing the phonons or lattice dynamics for a given crystal system consists in calculating a force-constant matrix to each of the neighbours of a given atom. In the embedded-atom semi-empirical method, the force constants matrix can be derived from the expression for the total energy E given by

$$
E=\sum_{i} F_{i}\left(\rho_{i}\right)+\frac{1}{2} \sum_{i \neq j} \phi(\mid R(i, j \mid)
$$

Here $F_{i}$ is the embedding energy of the atom $i$ which depends on the superposition of electronic charge densities $\rho_{i}=\sum_{j / i} \rho_{a i}(|i, j|)$ at site $\mathrm{i}$ from the surrounding atoms $\mathrm{j}$, and $\phi(|R(i, j)|)$, a screened pair potential is between atoms

$\mathrm{i}$ and $\mathrm{j}$. The functional forms are chosen for $\mathrm{F}_{\mathrm{i}}$ and $\phi_{i}$, and the parameters in these functions are determined by fitting to a limited number of bulk properties. We use in our calculation the simple exponentials for both the charge densities and the pair potentials.

In equation (2.30), If $\mathrm{R}(\mathrm{i}, \mathrm{j})$ is the vector between atoms $\mathrm{i}$ and $\mathrm{j}$, $R=|R(i, j)|$ is the

Vector length, $r_{o j}^{\alpha}=\frac{R_{o j}^{\alpha}}{\left|R_{o j}\right|}$ denotes the corresponding $\alpha$ component of the unit vector, then the force-constant tensor between atom ( $\mathrm{i}$ is atom at the origin $\mathrm{o}$ ) and another. Atom j can be written as [18 - 19].

$$
\begin{gathered}
\phi_{\alpha \beta}(0, j)=A(R)\left(\frac{R_{0 j}^{\alpha} R_{0 j}^{\beta}}{R_{0 j}^{2}}\right)+B(R)\left(\delta_{\alpha \beta}-\frac{R_{0 j}^{\alpha} R_{0 j}^{\beta}}{R_{0 j}^{2}}\right)+\sum_{l \neq 0} F^{\prime \prime}(\rho) \rho_{a}^{\prime}\left(R_{0 l}\right) \rho_{a}^{\prime}\left(R_{0 l}\right) \\
\left(\frac{R_{0 l}^{\alpha} R_{0 l}^{\beta}}{R_{0 l}^{2}}\right)
\end{gathered}
$$

Where $A(R)=\phi^{\prime \prime}\left(R_{0 j}\right)+F^{\prime}(\rho) \rho \rho_{a}^{\prime \prime}\left(R_{0 j}\right)$

$$
B(R)=\frac{\left(\phi^{\prime}\left(R_{0 j}\right)+F^{\prime}\left(R_{0 j}\right) \rho_{a}^{\prime}\left(R_{0 j}\right)\right)}{R_{0 j}}
$$

And $\delta_{\alpha \beta}$ is the Kronecker delta. 
The expressions (2.31) to (2.33) agree exactly with expressions given by Daw and Hatcher (1985) but differ slightly from the expression by Karimi and Mostoller (1992).

Equation (2.31) applies for monoatomic crystals with inversion symmetry. $\mathrm{A}(\mathrm{R})$ and $\mathrm{B}(\mathrm{R})$ in equation (2.31) represent the radial and tangential force constants, which involve the derivatives of the embedding function $\mathrm{F}$ and the electron density $\rho$ as well as the pair potential $\phi$.

In the calculation of the TB-SMA and the modified term contributions to the atomic force constants of atom $\mathrm{i}$, we take into account only those atoms located at distances less than $r_{c f}$ (cutoff distance) from atom $i$. In this paper, the atomic electron density $\rho\left(r_{i j}\right)$ is truncated at a specific cutoff distance. When the separation $\mathrm{r}_{\mathrm{ij}}$ between atoms $\mathrm{i}$ and $\mathrm{j}$ is larger than $r_{c f}$ that is $\left.\left(r_{i j}\right\rangle r_{c f}\right)$ , both $\rho\left(r_{i j}\right)$ and $\rho^{\prime}\left(r_{i j}\right)$ are equal to zero [Xiao-jun and Chang, (2013), Wilson and Riffe, (2012)].

The first two terms in Eq. (2.31) have exactly the form that occurs with central pair potentials alone, with radial and tangential force constants $A(R)$ and $\mathrm{B}(\mathrm{R})$.

However, these central force Constants involve derivatives of the embedding function $\mathrm{F}$ and the electron density $\rho$ as well as the pair potential $\phi$. The last term in Equation (2.31) goes beyond a central pair potential model, and represents the three-body contributions to the force-constants in the EAM [Aigbekaen, (2018),Hwang et al,(2010), Andrea, (2013), Mason, (2009)].

FORCE CONSTANT MATRIX, $\phi_{\alpha \beta}(0,1)$.

$\phi_{\alpha \beta}(0,1)=\left(\begin{array}{ccc}\alpha_{1} & \beta_{1} & \beta_{1} \\ \beta_{1} & \alpha_{1} & \beta_{1} \\ \beta_{1} & \beta_{1} & \alpha_{1}\end{array}\right)$.

FORCE CONSTANT MATRIX, $\phi_{\alpha \beta}(0,9)$.

$\phi_{\alpha \beta}(0,9)=\left(\begin{array}{ccc}\alpha_{2} & 0 & 0 \\ 0 & \beta_{2} & 0 \\ 0 & 0 & \beta_{2}\end{array}\right)$.

FORCE CONSTANT MATRIX, $\phi_{\alpha \beta}(0,15)$.

$\phi_{\alpha \beta}(0,15)=\left(\begin{array}{ccc}\alpha_{3} & \gamma_{3} & 0 \\ \gamma_{3} & \alpha_{3} & 0 \\ 0 & 0 & \beta_{3}\end{array}\right)$. 
FORCE CONSTANT MATRIX, $\phi_{\alpha \beta}(0,27)$.

$$
\phi_{\alpha \beta}(0,27)=\left(\begin{array}{lll}
\alpha_{4} & \gamma_{4} & \gamma_{4} \\
\gamma_{4} & \beta_{4} & \delta_{4} \\
\gamma_{4} & \delta_{4} & \beta_{4}
\end{array}\right)
$$

The dynamical matrix can written in $3 \times 3$ matrix form as shown below.

$$
\phi_{\alpha \beta}(0, l)=\left(\begin{array}{ccc}
\phi_{x x} & \phi_{x y} & \phi_{x z} \\
\phi_{y x} & \phi_{y y} & \phi_{y z} \\
\phi_{z x} & \phi_{z y} & \phi_{z z}
\end{array}\right) .
$$

Once the force constant matrices have been determined, the elements of the dynamical matrix can be evaluated up to fourth neighbours using:

$$
\begin{array}{r}
D_{\alpha \alpha}=\frac{1}{m_{A}}\left(A-8 \alpha_{1} \operatorname{Cos}\left(a q_{\alpha}\right) \operatorname{Cos}\left(a q_{\beta}\right) \operatorname{Cos}\left(a q_{\gamma}\right)-2 \alpha_{2} \operatorname{Cos}\left(2 a q_{\alpha}\right)-\right. \\
2 \beta_{2}\left(\operatorname{Cos}\left(2 a q_{\beta}\right)+\operatorname{Cos}\left(2 a q_{\gamma}\right)\right)-4 \alpha_{3} \operatorname{Cos}\left(2 a q_{\alpha}\right)\left(\operatorname{Cos}\left(2 a q_{\beta}\right)+\right. \\
\left.\operatorname{Cos}\left(2 a q_{\gamma}\right)\right)-4 \beta_{3} \operatorname{Cos}\left(2 a q_{\beta}\right) \operatorname{Cos}\left(2 a q_{\gamma}\right)- \\
8 \alpha_{4} \operatorname{Cos}\left(3 a q_{\alpha}\right) \operatorname{Cos}\left(a q_{\beta}\right) \operatorname{Cos}\left(\frac{a}{2} q_{\gamma}\right)- \\
\left.8 \beta_{4} \operatorname{Cos}\left(a q_{\alpha}\right)\left(\operatorname{Cos}\left(3 a q_{\beta}\right) \operatorname{Cos}\left(a q_{\gamma}\right)+\operatorname{Cos}\left(3 a q_{\gamma}\right) \operatorname{Cos}\left(a q_{\beta}\right)\right)\right)(2.75) \\
\text { Where } \alpha=x, y, z ; \alpha \neq \beta \neq \gamma \\
A=8 \alpha_{1}+2 \alpha_{2}+4 \beta_{2}+8 \alpha_{3}+4 \beta_{3}+8 \alpha_{4}+16 \beta_{4}
\end{array}
$$

$\alpha_{1}, \beta_{1}, \alpha_{2}, \beta_{2} \alpha_{3} \beta_{3}, \alpha_{4} \beta_{4}, \gamma_{1}, \gamma_{2}, \gamma_{3}, \gamma_{4}$, and $\delta_{3}$ are elements of the force constant matrix. are

$q_{\alpha}, q_{\beta}, q_{\gamma}$ the coordinates. $a$ is the lattice parameter of metals. A is often called the self-energy

term.

Also;

$D_{\alpha \beta}(\stackrel{\leftarrow}{q})=\frac{1}{m_{A}}\left(8 \beta_{1} \operatorname{Sin}\left(a q_{\alpha}\right) \operatorname{Sin}\left(a q_{\beta}\right) \operatorname{Cos}\left(a q_{\gamma}\right)+\right.$

$4 \gamma_{3} \operatorname{Sin}\left(2 a q_{\alpha}\right) \operatorname{Sin}\left(2 a q_{\beta}\right)+8 \gamma_{4} \operatorname{Cos}\left(a q_{z}\right)\left(\operatorname{Sin}\left(3 a q_{\alpha}\right) \operatorname{Sin}\left(a q_{\beta}\right)+\right.$

$\left.\left.\operatorname{Sin}\left(3 a q_{\beta}\right) \operatorname{Sin}\left(a q_{\alpha}\right)\right)+8 \delta_{4} \operatorname{Sin}\left(a q_{\alpha}\right) \operatorname{Sin}\left(a q_{\beta}\right) \operatorname{Cos}\left(3 a q_{\gamma}\right)\right)$ 
Where $(\alpha \neq \beta \neq \delta)$.

where; $m_{A}$ denotes the atomic mass of the given metal atom.

Where; $\mathrm{D}(\mathrm{q})$ is often termed the dynamical matrix, is the Eigen value. Once the Eigen values are known up to fourth order, we plot the Eigen values against the K-points, to get the phonon dispersion curve of the crystal.

\subsection{Phonon thermodynamic properties of alkali metals}

Once the phonon spectrum has been obtained over the entire Brillouin zone, one can now calculate and obtain the phonon free energy, $\Delta \mathrm{F}$, the internal energy, $\Delta \mathrm{E}$, the constant-volume specific heat, $\mathrm{C}_{\mathrm{V}}$, at zero pressure and the entropy, $S$.

The Expressions used for phonon free energy, the internal energy, the constant-volume specific heat and the entropy are given as follows :

\section{HELMHOLTZ FREE ENERGY, $\triangle F$.}

$$
\Delta \mathrm{F}=3 \mathrm{Nnk}_{\mathrm{B}} \mathrm{T} \int_{0}^{\omega_{L}} \operatorname{In}\left\{2 \sinh \left(\frac{\hbar \omega}{2 T K_{B}}\right)\right\} g(\omega) d \omega
$$

2. INTERNAL ENERGY, $\triangle E$.

$$
\Delta \mathrm{E}=3 \mathrm{Nn}_{2}^{\frac{\hbar}{2}} \int_{0}^{\omega_{L}} \omega \operatorname{coth}\left(\frac{\hbar \omega}{2 T K_{B}}\right) g(\omega) d \omega
$$

3. CONSTANT-VOLUME SPECIFIC HEAT, $\mathrm{C}_{\mathrm{v}}$.

$$
\mathrm{C}_{\mathrm{V}}=3 \mathrm{Nnk}_{\mathrm{B}} \int_{0}^{\omega_{L}}\left(\frac{\hbar \omega}{2 T K_{B}}\right)^{2} \operatorname{csch}^{2}\left(\frac{\hbar \omega}{2 T K_{B}}\right) g(\omega) d \omega
$$

\section{ENTROPY, $\mathrm{S}$.}

$\mathrm{S}=3 \mathrm{Nnk}_{\mathrm{B}} \int_{0}^{\omega_{L}}\left[\frac{\hbar \omega}{2 T K_{B}} \operatorname{coth} \frac{\hbar \omega}{2 T K_{B}}-\operatorname{In}\left\{2 \sinh \left(\frac{\hbar \omega}{2 T K_{B}}\right)\right\}\right] X g(\omega) d \omega$

where $K_{B}$ is the Boltzmann constant, $n$ is the number of atoms per unit cell, $N$ is the number of unit cells, $\omega_{\mathrm{L}}$ is the largest phonon frequency, $\omega$ is the phonon frequency and $\mathrm{g}(\omega)$ is the normalized phonon density of states with $\int_{0}^{\omega_{L}} g(\omega) d \omega=1, \Delta \omega$ is the frequency width. 


\section{Results and discussion}

Table 1: TB-SMA parameters and other input parameters

\begin{tabular}{|c|c|c|c|c|c|}
\hline $\begin{array}{l}\text { PARAMETER } \\
\text { S }\end{array}$ & $\mathbf{L i}$ & $\mathbf{N a}$ & $\bar{K}$ & $\mathbf{R b}$ & Cs \\
\hline $\mathbf{N}$ & 0.2836 & 0.2954 & 0.3537 & 0.3087 & 0.3163 \\
\hline $\mathbf{P}$ & 46.4200 & 48.4470 & 42.8100 & 45.8400 & 38.2970 \\
\hline q & 0.2836 & 0.2821 & 0.2909 & 0.4247 & 0.4237 \\
\hline$\xi(\mathrm{eV})$ & 0.5798 & 0.3961 & 0.3325 & 0.3041 & 0.2874 \\
\hline A & $1.2524 \mathrm{E}-03$ & 8.1485E-04 & 7.9876E-04 & 9.9593E-04 & 1.1243E-03 \\
\hline $\mathrm{U}_{0}(\mathrm{eV})$ & 1.6300 & 1.1130 & 0.9340 & 0.8520 & 0.8040 \\
\hline$E_{I V}^{F}$ & 0.4800 & 0.3400 & 0.3400 & 0.2700 & 0.2600 \\
\hline $\mathbf{a}(\stackrel{\AA}{\mathbf{A}})$ & 3.5092 & 4.2906 & 5.3200 & 5.7000 & 6.1400 \\
\hline $\mathbf{R}(\AA)$ & 3.0391 & 4.2906 & 8.8222 & 9.4524 & 10.1820 \\
\hline $\mathrm{C}_{11}\left(\mathrm{erg} / \mathrm{cm}^{2}\right)$ & $0.1440 \mathrm{E}+12$ & $0.08160 \mathrm{E}+12$ & $0.04150 E+12$ & $0.03120 \mathrm{E}+12$ & $0.02470 \mathrm{E}+12$ \\
\hline $\mathrm{C}_{12}\left(\mathrm{erg} / \mathrm{cm}^{2}\right)$ & $0.1210 \mathrm{E}+12$ & $0.06790 E+12$ & $0.03400 \mathrm{E}+12$ & $0.02620 \mathrm{E}+12$ & $0.02060 \mathrm{E}+12$ \\
\hline $\mathrm{C}_{44}\left(\mathrm{erg} / \mathrm{cm}^{2}\right)$ & $0.1070 \mathrm{E}+12$ & $0.0570 \mathrm{E}+12$ & $0.02840 E+12$ & $0.01860 \mathrm{E}+12$ & $0.01480 \mathrm{E}+12$ \\
\hline$B_{O}\left(\mathrm{erg} / \mathrm{cm}^{2}\right)$ & $0.1160 E+12$ & $0.0680 E+12$ & $0.0320 E+12$ & $0.0310 E+12$ & $0.0200 E+12$ \\
\hline $\mathbf{P}$ & 8.0000 & 11.4184 & 11.1455 & 7.7933 & 7.8136 \\
\hline $\boldsymbol{\rho}^{\prime}(\mathbf{r})(\mathbf{1} /(\AA ̊ \cap))$ & -1.4931 & -3.7023 & -2.9736 & -2.4337 & -2.2623 \\
\hline$\rho^{\prime \prime}(\mathbf{r})\left(\mathbf{1} /(\AA)^{2}\right)$ & 0.27867 & 0.8761 & 0.5834 & 0.6197 & $\mathbf{0 . 5 3 3 7}$ \\
\hline$\phi(\mathbf{r})$ & 2.5049E-03 & 9.1904E-23 & $-1.5644 \mathrm{E}-20$ & 1.2198E-21 & 1.3674E-18 \\
\hline$\phi^{\prime}(\mathbf{r})$ & $-3.8261 \mathrm{E}-02$ & $-1.1904 \mathrm{E}-21$ & $-1.4536 E-20$ & $-1.1327 E-20$ & $-9.8486 E-18$ \\
\hline$\phi^{\prime \prime}(\mathbf{r})$ & 0.5844 & $1.5623 \mathrm{E}-20$ & $1.3507 \mathrm{E}-18$ & 1.0519E-19 & 7.0946E-17 \\
\hline $\mathbf{F}(\rho)$ & -1.04573 & -0.9101 & -8.9099E-01 & -0.6243 & -0.6014 \\
\hline $\mathbf{F}^{\prime}(\rho)$ & -3.7071E-02 & $-1.6050 E-02$ & $-1.9415 E-02$ & -1.8739E-02 & $-1.8434 E-02$ \\
\hline $\mathbf{F}^{\prime /}(\rho)$ & 3.3197E-02 & 6.7517E-04 & 7.7303E-04 & 1.2595E-03 & 1.2214E-03 \\
\hline $\mathbf{A}(\mathbf{R})\left(\mathrm{eV} /\left(\AA \AA^{\mathbf{A}}\right)^{2}\right)$ & 0.5741 & $-1.4061 E-02$ & $-1.1327 E-02$ & $-1.1612 E-02$ & $-9.8380 E-03$ \\
\hline $\mathbf{B}(\mathbf{R})\left(\mathrm{eV} /(\stackrel{\AA}{\mathrm{A}})^{2}\right)$ & $5.6230 \mathrm{E}-03$ & 8.3513E-03 & $6.5440 \mathrm{E}-03$ & 4.8246E-03 & 4.0958E-03 \\
\hline
\end{tabular}

Table 2: Force-Constants for alkali metals

\begin{tabular}{|l|l|l|l|l|l|}
\hline $\begin{array}{l}\text { FORCE- } \\
\begin{array}{l}\text { CONSTAN } \\
\text { T }(\mathbf{d y n} / \mathbf{c m})\end{array}\end{array}$ & METALS & $\mathrm{Li}$ & $\mathrm{K}$ & $\mathrm{Rb}$ & $\mathrm{Cs}$ \\
\hline$\alpha_{1}$ & 452.4276 & 240.9389 & 136.7971 & 114.7673 & 85.2672 \\
\hline$\alpha_{2}$ & 261.8110 & 185.5323 & 146.9431 & 125.8242 & 107.2560 \\
\hline$\alpha_{3}$ & 203.6961 & 134.7058 & 105.7361 & 83.3440 & 70.9171 \\
\hline$\alpha_{4}$ & 201.5032 & 127.8249 & 101.6976 & 74.7862 & 63.7734 \\
\hline$\beta_{1}$ & 362.4534 & 169.6712 & 56.6538 & 60.6007 & 36.6637 \\
\hline$\beta_{2}$ & 284.3606 & 195.6712 & 152.1798 & 129.7258 & 110.0003 \\
\hline$\beta_{3}$ & 212.0983 & 139.1377 & 108.0112 & 85.2570 & 72.3134 \\
\hline
\end{tabular}




\begin{tabular}{|l|l|l|l|l|l|}
\hline$\beta_{4}$ & 211.5645 & 132.9763 & 104.3695 & 76.9268 & 65.3373 \\
\hline$\gamma_{3}$ & -8.4022 & -4.4319 & -2.2751 & -1.9130 & -1.3964 \\
\hline$\gamma_{4}$ & -3.7730 & -1.9318 & -1.0020 & -0.8027 & -0.5865 \\
\hline$\delta_{4}$ & -1.2577 & -0.6439 & -0.3340 & -0.2675 & -0.1955 \\
\hline
\end{tabular}

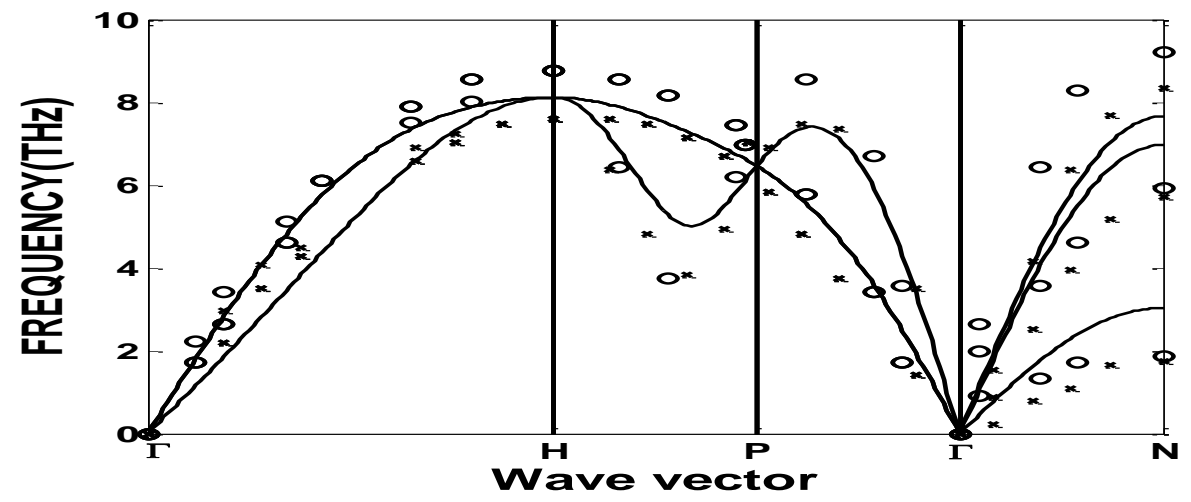

Fig.1: Phonon dispersion curves for $\mathrm{Li}$

- TB-SMA.

X EAM.

O EXPERIMENT.

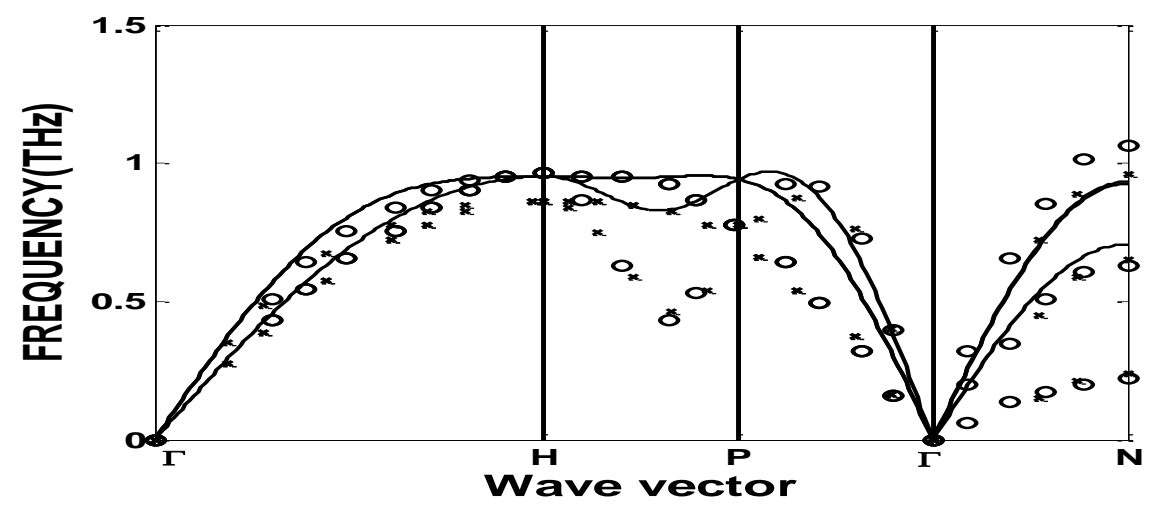

Fig. 2: Phonon dispersion curves for Cs.

TB-SMA.

X EAM.

O EXPERIMENT. 


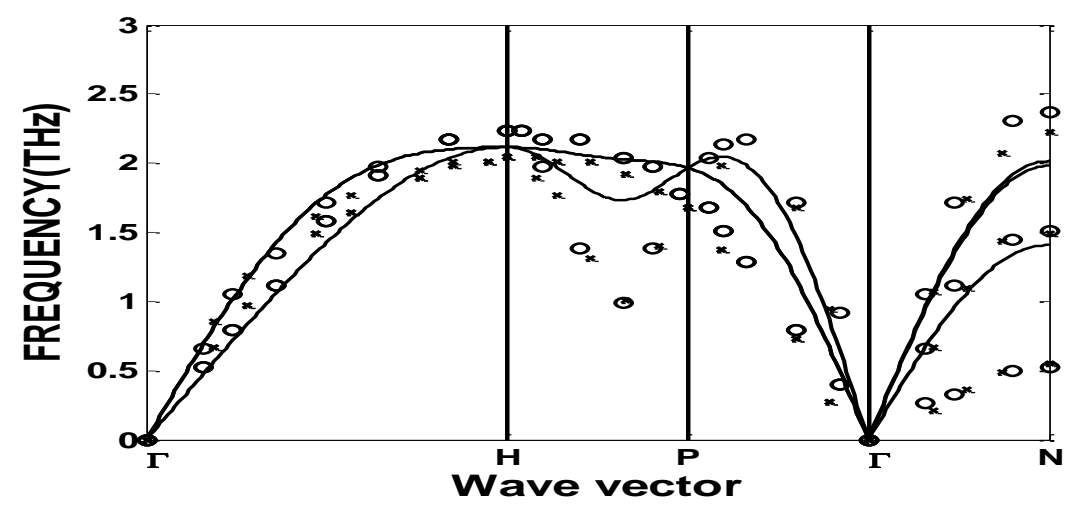

Fig. 3: Phonon dispersion curves for K.

X EAM.

O EXPERIMENT.

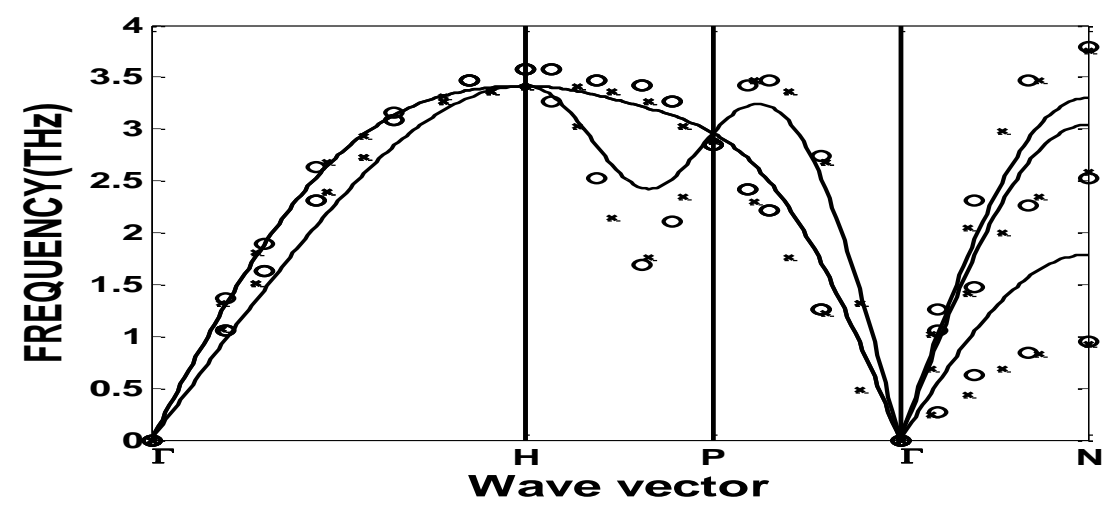

Fig. 4: Phonon dispersion curves for Na.

X EAM.

O EXPERIMENT. 


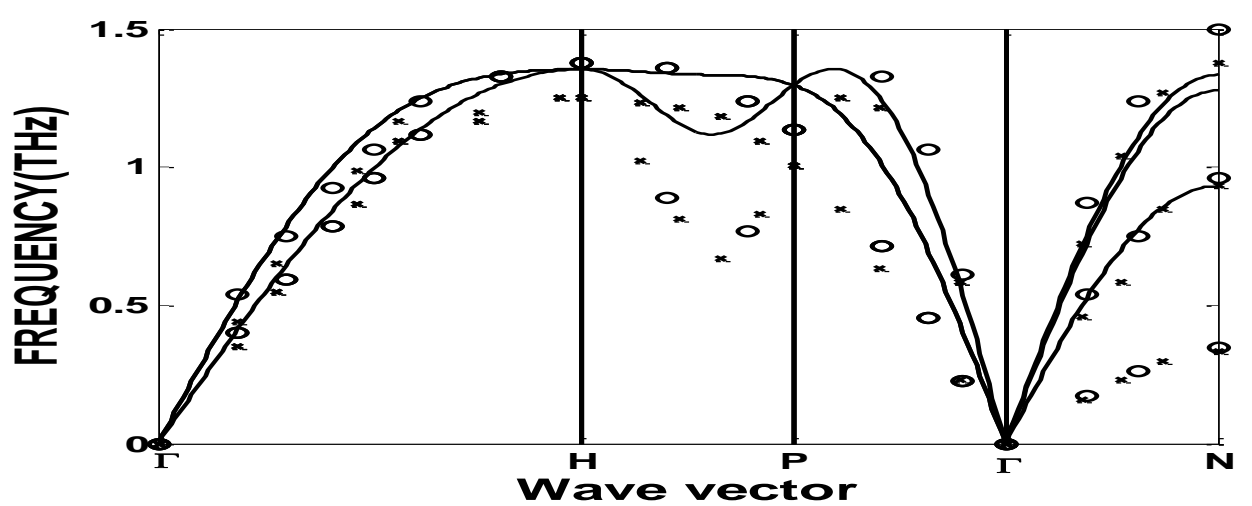

Fig.5: Phonon dispersion curves for $\mathrm{Rb}$.

- TB-SMA.

X EAM.

O EXPERIMENT.

THERMODYNAMICS PROPERTIES
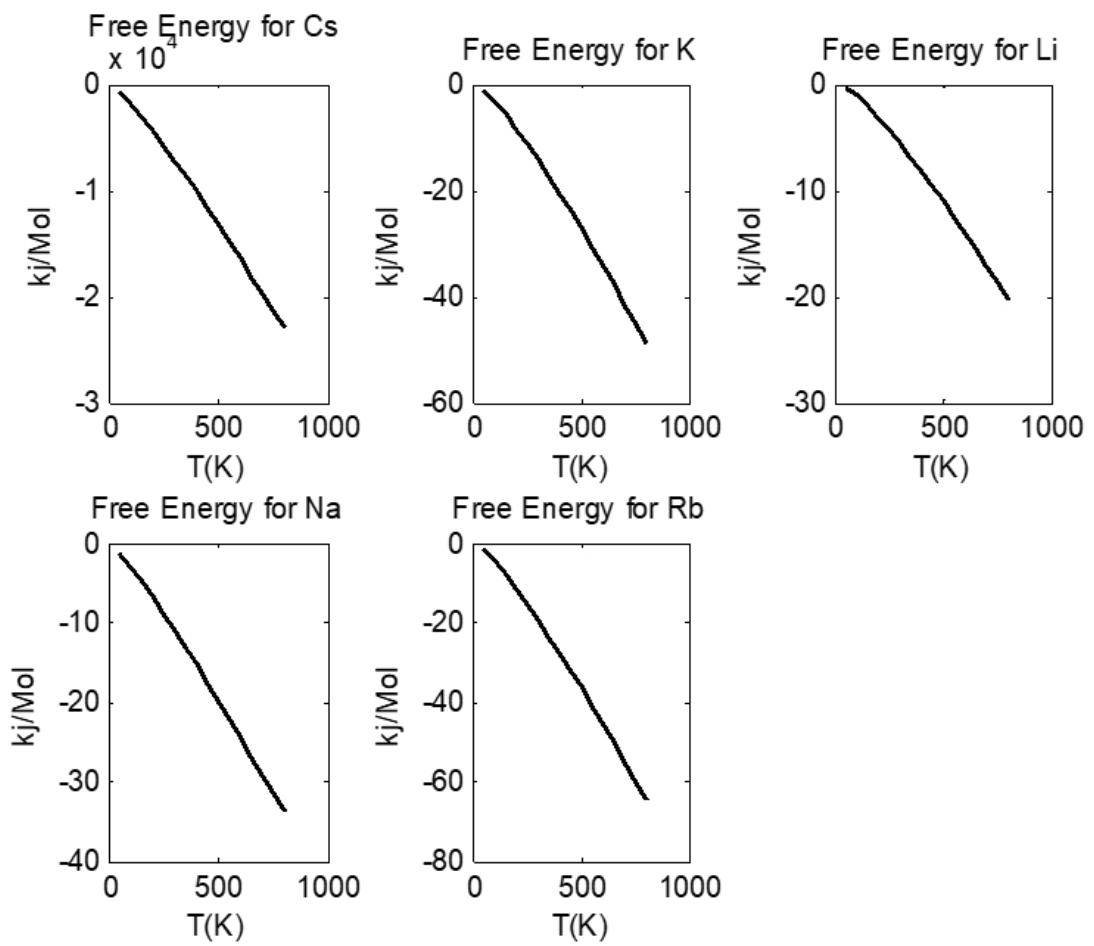

Fig. 6: Showing the free energy of Alkali Metals 

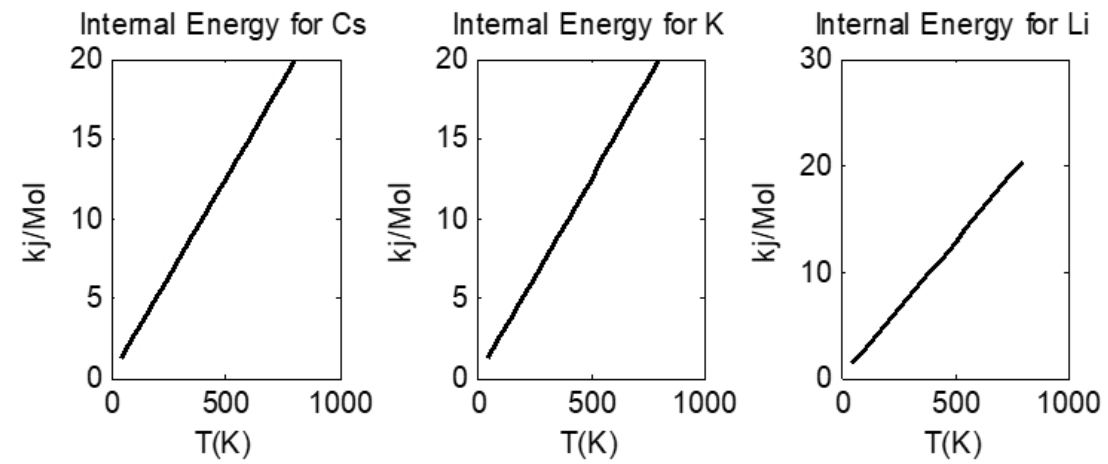

Intemal Energy for $\mathrm{Na}$
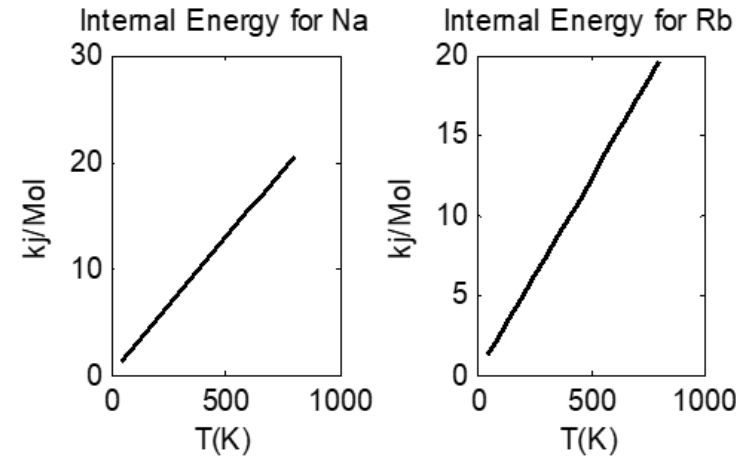

Fig.7: Showing the Internal energy of Alkali Metals.
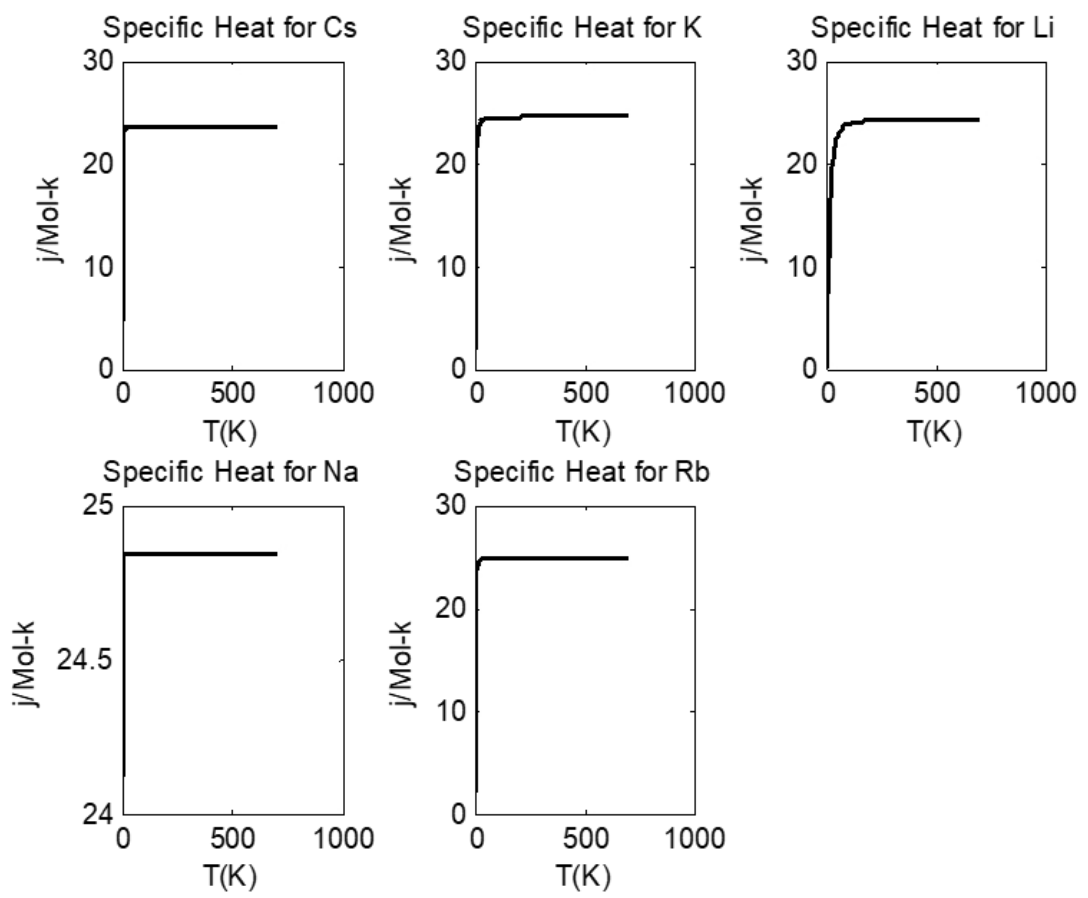

Fig.8: Showing the Specific Heat of Alkali Metals. 

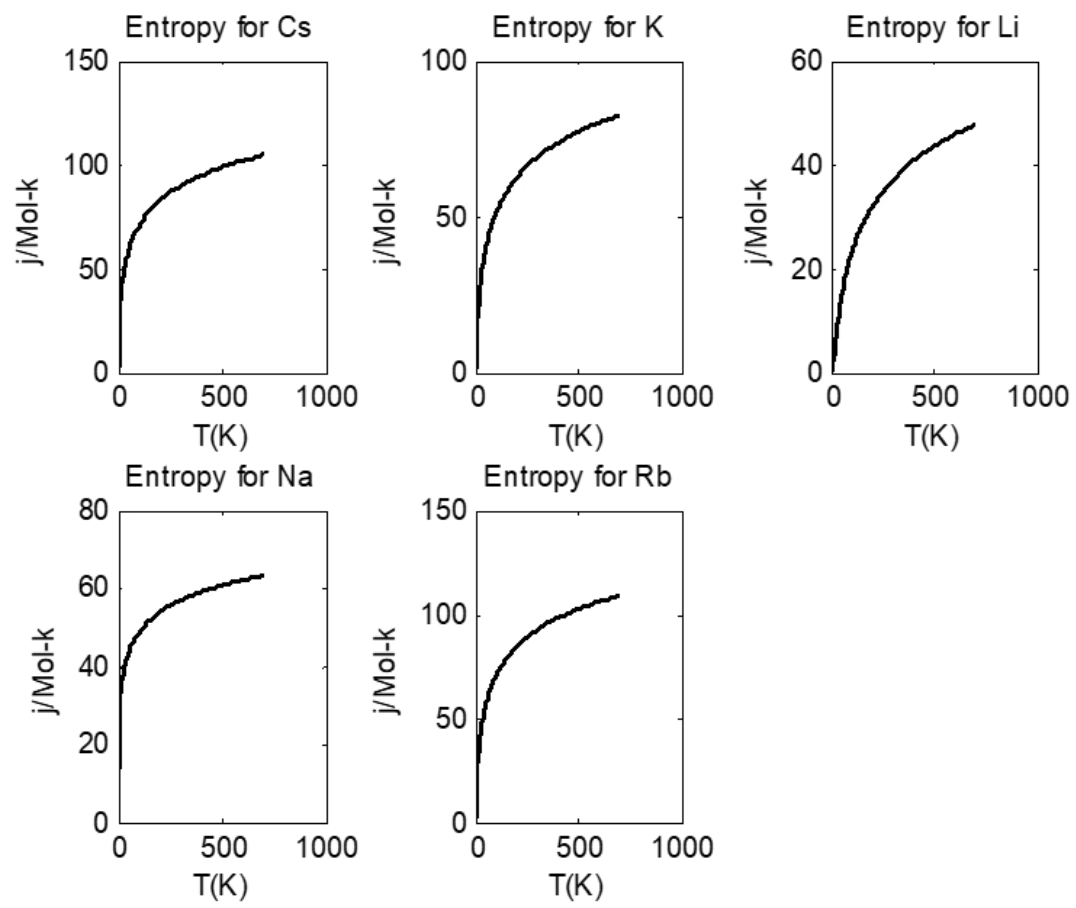

Fig.9: Showing the Entropy of Alkali Metals.

\section{Discussion}

We have performed the simulations for the Phonon dispersion curves along the high symmetry directions $[0,0,0],[\xi, \xi, \xi]$ and $[0, \xi, \xi]$ for the alkali metals. The notation $\Gamma, \mathrm{H}, \mathrm{P}$, and $\mathrm{N}$ are the special points in the first Brillouin zone of the bcc lattice, $\mathrm{L}$ and $\mathrm{T}$ are the longitudinal mode and transverse mode respectively. The results are shown in Fig. 1 to Fig.5

\section{LITHIUM, Li.}

A look at fig. 1 shows that there is an agreement between TB-SMA phonon dispersion Curve, EAM phonon dispersion curve and experimental phonon dispersion Curve along the symmetry directions. TB-SMA phonon dispersion Curve deviated slightly in the $[0, \xi, \xi]$ direction, there was an intersection of the LM and TM of the experimental phonon dispersion Curve, EAM phonon dispersion curve at a point in $\Gamma$ to $\mathrm{H}$ points and in $\mathrm{H}$ to $\mathrm{P}$ of $[\xi$, $\xi$, $\xi$ ] direction, TB-SMA dispersion Curve agree with experimental dispersion Curve but in $\mathrm{P}$ to $\Gamma$ points in the $[\xi, \xi, \xi]$ direction, TB-SMA phonon dispersion Curve agree with experimental phonon dispersion Curve and in $\Gamma$ to $\mathrm{N}$ points in the $[\xi, \xi, 0]$ direction, TB-SMA phonon dispersion Curve, EAM phonon dispersion curve are in excellent agreement with the experimental phonon dispersion Curve. 


\section{CAESIUM, Cs.}

A close study of fig. 2 reveals that there is close agreement between TB-SMA phonon dispersion Curve, EAM phonon dispersion curve and experimental phonon dispersion Curve for caesium for some wave vectors in all the three principal symmetry directions. For phonon dispersion Curve there is a little discrepancy between TB-SMA phonon dispersion Curve, EAM phonon dispersion curve and experimental dispersion Curve in the L modes and $\mathrm{T}$ modes of $[0, \xi, \xi]$ direction in $\Gamma$ to $\mathrm{H}$ points, TB-SMA phonon dispersion Curve and EAM phonon dispersion curve are in agreement with the experimental phonon dispersion Curve, and in $\mathrm{H}$ to $\mathrm{P}$ points of $[\xi, \xi, \xi]$ direction, TB-SMA phonon dispersion Curve and EAM phonon dispersion curve are in agreement with the experimental phonon dispersion Curve. In $\mathrm{P}$ to $\Gamma$ points in the $[\xi, \xi, \xi]$ direction, TB-SMA phonon dispersion Curve and EAM phonon dispersion curve are in agreement with the experimental phonon dispersion Curve, and in $\Gamma$ to $\mathrm{N}$ points in the $[\xi, \xi, 0]$ direction, TB-SMA phonon dispersion Curve, and EAM phonon dispersion curve are in agreement with the experimental phonon dispersion Curve.

\section{POTASSIUM, $\mathrm{K}$.}

A close study of fig. 3 shows that there is a slight agreement between TB-SMA phonon dispersion Curve, EAM phonon dispersion curve and experimental dispersion Curve along the symmetry directions. In the $[0, \xi, \xi]$ direction, of $\Gamma$ to $H$ points of TB-SMA agrees with experimental Curve, while EAM phonon dispersion curve agree a great deal with experimental Curve, and in $\mathrm{H}$ to $\mathrm{P}$ points of $[\xi, \xi, \xi]$ direction, TB-SMA agrees with experimental Curve, while EAM phonon dispersion curve agree with experimental Curve. In $\mathrm{P}$ to $\Gamma$ points, in $[\xi, \xi, \xi]$ direction, TB-SMA agrees with experimental Curves, while EAM phonon dispersion curve agree a great deal with experimental Curve, and in $\Gamma$ to $\mathrm{N}$ points in the $[\xi, \xi, 0]$ direction, TB-SMA disagree with EAM phonon dispersion curve and experimental dispersion curve.

\section{SODIUM, Na.}

A critical study of fig.4 reveals that there is close agreement between the TB-SMA phonon dispersion Curve, EAM phonon dispersion curve and experimental phonon dispersion Curve for sodium for almost all wave vectors in all the three principal symmetry directions. For this study there is a little discrepancy between the TB-SMA phonon dispersion Curve, EAM phonon dispersion curve and experimental phonon dispersion Curve in the $\mathrm{L}$ modes and $\mathrm{T}$ modes of $[0, \xi, \xi]$ direction in the $\Gamma$ to $\mathrm{H}$ points and $\mathrm{H}$ to $\mathrm{P}$ of $[\xi, \xi, \xi]$ direction, but its phonon dispersion curve agrees in the $\mathrm{P}$ to $\Gamma$ points in the $[\xi$, $\xi, \xi]$ direction, and also in $\Gamma$ to $\mathrm{N}$ point in the $[\xi, \xi, 0]$ direction. 


\section{RUBIDIUM, Rb.}

A look at fig. 5 shows that there is an agreement between TB-SMA phonon dispersion Curve, EAM phonon dispersion Curve and experimental phonons dispersion Curve along the symmetry directions. TB-SMA phonon dispersion Curve and EAM phonon dispersion Curve agrees with experimental phonon dispersion Curve in the $[0, \xi, \xi]$ direction i.e. $(\Gamma$ to $\mathrm{H})$ and in $\mathrm{H}$ to $\mathrm{P}$ of $[\xi, \xi, \xi]$ direction, TB-SMA phonon dispersion Curve and EAM phonon dispersion Curve are in agreement with experimental phonon dispersion Curve but at $\mathrm{P}$ to $\Gamma$ points in the $[\xi, \xi, \xi]$ direction, TB-SMA phonon dispersion Curve and EAM phonon dispersion Curve are in agreement with the experimental dispersion curve and in $\Gamma$ to $\mathrm{N}$ points in the $[\xi, \xi, 0]$ direction, TB-SMA phonon dispersion Curve, and EAM phonon dispersion curve and are in agreement with experimental phonon dispersion Curve.

The thermodynamics properties of the alkali metals were calculated using equations (2.78) to (2.81) and plotted as shown in Fig.6 to Fig.9.

\section{Conclusion}

The five unknown parameters in the TB-SMA Scheme have been determined in this paper, But the parameter $\mathrm{n}$ is not constant for all the alkali metals, as usually assumed in literature. The use of the constant parameter, $\mathrm{n}$ $=1 / 2$, by Cleri and Rosato (1993) in predicting the phonon dispersion curves for alkali metals was unsuccessful, despite adopting larger sets of free parameters and larger interaction ranges of the atoms.

The phonon dispersion curves obtained from TB-SMA have been reported in this study. For TB-SMA model (first nearest neighbour model to the fourth next nearest neighbour model), Sodium metal was found to give better agreement with experiment than the other alkali metals.

The results obtained in this paper, although fairly satisfactory; do not show the complete fine Structure characteristics found in the experimental phonon dispersion curves.

The thermodynamics properties of the alkali metals were calculated and plotted and the curves were in excellent agreement with those shown in literature.

This study has shown that the phonon dispersion of alkali can be deduce, by consistently varying the TB-SMA parameter $\mathrm{n}$ instead of using a fixed value of $n(1 / 2$ or $2 / 3)$ as in literature.

\section{References:}

1. Daw M. S.and Baskes M.I.(1984): Embedded - Atom Method Derivation and Application to Impurities, Surface and Other Defects in Metal .Phys. Rev. B 29, 6443 - 6453. 
2. Cleri, F and Rosato, V (1993). Tight -Binding Potentials for Transition Metals and Alloys. Phys. Rev. B 48, 22 -33.

3. Daw, M S and Hatcher, R D (1985). Application of The Embedded Atom Method To Phonons In Transition Metals, Solid State Communications, Vol.56, No.8,697-699.

4. Ozdemir K., Erbay A., Kilic H., Cagin T. and Tomak M. (2008): Molecular Dynamics study of $\mathrm{Cu}-\mathrm{Pd}$ ordered alloys. J. of Achievements in Materials and Manufacturing Engineering, Vol. 31, $41-46$.

5. Jun C. and Jian-Sheng W. (2002): Modeling generalized stacking faults in $\mathrm{Au}$ Using the tight- binding potential combined with a simulated annealing method. Eur.Phys.J. B 28, 45-48.

6. Idiodi, J O A (2000). Tight-Binding Potentials for FCC Metals. J. Nig. Ass Math. Physics, Vol. 4, 31-39.

7. Guevara, J, Llois, A M and Weissmann, M (1995). Model Potential Based on Tight -Binding Total - Energy Calculations for Transition Metal Systems. Phys. B52, 11509 - 11516.

8. Carlsson, A E (1991). Angular Forces in Group -VI Transition Metals: Application to W (100). Phys. Rev. B 44, 6590 -6597.

9. Johnson R. A. (1988): Analytic Nearest -Neighbour Model for FCC Metals.Phys. Rev. B 37, 3924 -3931.

10. Idiodi J.O.A (2000): Tight-Binding Potentials for FCC Metals. J. Nig. Ass Math. Physics, Vol. 4, 31-39.

11. Mehl M .J .and Papaconstantopoulos D. A. (1996): Applications of a Tight-Binding Total-Energy Method for Transition and Noble Metals: Elastic Constants, Vacancies and Surfaces of Monatomic Metals "Phys. Rev. B 54, 4519 - 4530.

12. Karolewski, E (2001). Tight- binding potentials for Sputtering Simulations with fcc and bcc Metals. Radiation Effects and Defects in solids Vol.153, 235-239.

13. Idiodi J.O.A .and Aghemenloh E. (1998): On the Problem of Low Surface Energies Within the Embedded Atom Method .J. Nig. Assoc. Math .Physics Vol. 2, 285 -296.

14. Willaime F. and Massobrio C. (1989): Temperature - Induced hcp-bcc Phase Transformation in Zirconium: A Lattice and Molecular Dynamics Study Based on an N -Body Potential. Phys. Rev. Lett. 63, $2244-2247$.

15. Sigalas M. M. and Papaconstantopoulos D. A.(1994) : Transferable Total-Energy Parameterization for Metals: Applications to ElasticConstant Determination. Phys.Rev. B49, 1574 - 1579. 
16. Strich I., Car R. and Parrinello M. (1991): Amorphous Silicon Studied by ab Initio Molecular Dynamics Preparation. Structure and Properties: Phys,Rev.B 44.11092-110910.

17. Youhong Li, Donald J.Siegel, James B.Adams and Xiang - Yang Liu (2003): Embedded -atom-method tantalum potential developed by the force-Matching method. Phys. Rev. B 67, 125101-125108.

18. Karimi, M and Mostoller, M (1992). Embedded-atom-method of (110) surface relaxation and bulk vibration in Pb. Phys. Rev. B 45, $6289-$ 6292.

19. Sutton A. P ;Finnis M. W, Pettifor D.G and Ohta .Y (1988): TightBinding Bond Model. J. Phys. C; Solid State Phys, Vol. 21,35-66.

20. Xiao-jun, Z, and Chang Le, C (2013). Pressure Dependence of Phonon Dispersion in Bcc Tungsten, Chinese Journal of Physics, Vol. 51, No.2,359-364.

21. Wilson, R B and Riffe, D M, (2012). An Embedded-Atom-Method Model for Alkali-Metal Vibrations. J.Phys., Condens. Matter 24, 335401,1-6.

22. Aigbekaen E.E. (2018): Phonon Dispersion of Some BBC Metals, Using the TB-SMA of Cohesion, Ph.D Thesis, University of Benin, Benin City.

23. Shun-Fa Hwang, Yi-Hung Li, and Zheng-Han Han Hong (2010): Critical conditions of Epitaxy, Mixing and Sputtering for Cluster Deposition on Si substrate. Recent Research in Mechanics, 60-65. Andrea, D C (2013). Ab initio phonon dispersions of transition and noble metals;

24. Mason D. R, Foulkes W. M. C, Sutton A. P (2009): A Simple model for large-Scale simulations of fcc metals with explicit treatment of electrons. Philosophical Magazine Letters, Vol. 90, 51-60. 